\title{
Short term outcomes of helical tomotherapy during concurrent chemoradiotherapy for advanced cervical cancer
}

\author{
YASUSHI MABUCHI, YOSHIHIRO TAKIGUCHI, TAMAKI YAHATA, MIKA MIZOGUCHI, \\ NORIYUKI SASAKI, NAMI OTA, SAWAKO MINAMI and KAZUHIKO INO \\ Department of Obstetrics and Gynecology, Wakayama Medical University, \\ School of Medicine, Wakayama, Wakayama 641-0012, Japan
}

Received June 20, 2018; Accepted November 14, 2018

DOI: $10.3892 /$ mco.2019.1806

\begin{abstract}
The aim of the present study was to clarify the feasibility and efficacy of helical tomotherapy during concurrent chemoradiotherapy for treating cervical cancer. The medical records of 13 patients who underwent oncurrent chemoradiotherapy using helical tomotherapy for cervical cancer at Wakayama Medical University Hospital between 2013 and 2015 were retrospectively reviewed. A total of 15 patients who underwent oncurrent chemoradiotherapy using conventional radiotherapy (CRT) between 2008 and 2013 at our institution were also examined for comparison. The median age of patients treated with helical tomotherapy was 60 (range, 35-71), and the median age of patients treated with CRT was 57 (range, 43-77). The median follow-up period was 27 months (range, 3-46) in the tomotherapy group and 35 months (range, 7-88) in the CRT group. The frequency of G3/4 thrombocytopenia in the tomotherapy group was significantly higher than that in the CRT group $(\mathrm{P}=0.049)$. However, the platelet count spontaneously recovered without transfusion. There were no significant differences between the groups in terms of frequency of G3/4 neutropenia, diarrhea or late intestine injury. The rate of complete response in the tomotherapy group and the CRT group was 84.6 and $73.3 \%$, respectively, and there was no significant difference in the response rate between the groups. There were no significant differences in the progression-free survival or progression-free rate in the irradiation field between the groups. Adverse events from concurrent chemoradiotherapy using helical tomotherapy were acceptable and clinically controllable. The present results suggest that helical tomotherapy is efficient during concurrent chemoradiotherapy for treatment of advanced cervical cancer.
\end{abstract}

Correspondence to: Dr Yasushi Mabuchi, Department of Obstetrics and Gynecology, Wakayama Medical University, School of Medicine, 811-1 Kimiidera, Wakayama, Wakayama 641-0012, Japan

E-mail: booyan@wakayama-med.ac.jp

Key words: helical tomotherapy, concurrent chemoradiotherapy, cervical cancer, radiation therapy, outcome

\section{Introduction}

Locally advanced cervical cancer without distant metastasis [International Federation of Gynecology and Obstetrics (FIGO) stage IIB, IIIA, IIIB or IVA] is usually treated with definitive pelvic radiation and concurrent chemotherapy. However, increased acute toxicity involving hematological, gastrointestinal or genitourinary systems by the addition of concurrent chemotherapy to radiotherapy has been reported (1-3). In Kirwan's meta-analysis, late grade 3 or 4 toxicity (cystitis, proctitis, or intestinal obstruction) was observed in $23.3 \%$ in the chemoradiotherapy group (4).

According to the NCCN clinical practice guidelines for cervical cancer, version 1.2017, intensity-modulated radiation therapy (IMRT) and similar highly conformed methods of dose delivery may be helpful in minimizing the dose to the bowel and other critical structures in the IMRT post-hysterectomy setting and in treating the para-aortic nodes when necessary. In the Japan Society of Gynecologic Oncology (JSGO) guidelines 2017 for the treatment of uterine cervical cancer, it is stated that the dose to the bowel, bladder, or bone marrow may be reduced by using IMRT compared with conventional radiotherapy, which may lead to a lower rate of complications in the post-hysterectomy setting. According to the Japanese guidelines, appropriate treatment planning is necessary considering the dose limitation to normal organs and the volume change in the bladder or rectum during IMRT. Gandhi et al (5) previously reported the toxicity and clinical outcome in patients with locally advanced cervical cancer treated with whole pelvic conventional radiation therapy and IMRT, and concluded that definitive whole pelvic IMRT was associated with significantly less toxicity compared with whole pelvic conventional radiation therapy and had a comparable clinical outcome.

Helical tomotherapy is a novel radiotherapy method for IMRT combined with image guided radiation therapy based on computed tomography. Using this method, high conformity and critical organ sparing ability are expected. However, there are still a small number of institutions practically using helical tomotherapy. Although IMRT for the treatment of uterine cervical cancer has been described in practical guidelines from the USA and Japan as well as in the reports mentioned above, there are only a few reports 
on helical tomotherapy as definitive radiotherapy for cervical cancer. Herein, we analyzed the feasibility and efficacy of helical tomotherapy during concurrent chemoradiotherapy for cervical cancer.

\section{Materials and methods}

We retrospectively reviewed the medical records of 13 patients who underwent concurrent chemoradiotherapy using helical tomotherapy (Tomo HD system; Accuray, Inc., Madison, WI, USA) for cervical cancer at Wakayama Medical University Hospital between 2013 and 2015. More than 2 years have passed since the beginning of each patient's treatment. We also examined 15 patients who underwent concurrent chemoradiotherapy using conventional radiotherapy (CRT) between 2008 and 2013 at our institution for comparison. We included all patients who underwent concurrent chemoradiotherapy in the period. Patients undergoing hysterectomy before concurrent chemoradiotherapy were excluded. The data of age, clinical stage, histological subtype, tumor size, and chemotherapy/radiotherapy regimen were extracted from patients' files and analyzed. Written informed consent was obtained from the patients, and patient anonymity was preserved.

Radiotherapy. The radiotherapy was composed of external beam radiotherapy (EBRT) and high dose rate intracavity brachytherapy (ICBT). We used 3-dimensional conformal radiotherapy based on $\mathrm{CT}$ planning as the conventional radiotherapy. In the CRT group, 4-field technique was performed while anteroposterior- posteroanterior technique was used in center split. The prescribed dose for PTV, received by $95 \%$ of the volume, was 45-50 Gy for the whole pelvis and 5-10 Gy for para-aortic lymph nodes in the tomotherapy group. The dose of ICBT was $11.5 \mathrm{~Gy}$ in the tomotherapy group. On the other hand, the dose of irradiation was 45-50 Gy, for the whole pelvis including the center split irradiation and 12-14 Gy for para-aortic lymph nodes, and the dose of ICBT was 23 Gy at point A in the CRT group. The distribution of irradiation dose in the CRT group was determined in compliance with General Rules for Clinical and Pathological Study of Uterine Cervical Cancer in Japan (1997). Para-aortic irradiation was carried out for patients with common iliac lymph node metastases in both groups. The biologically effective dose was 71.2-79.2 Gy in the tomotherapy group and 72.2-72.3 Gy in the conventional radiotherapy group. The cumulative equivalent dose (EQD2) was 59.3-66 Gy in the tomotherapy group, and 60.2-60.3 Gy at point $\mathrm{A}$ in the conventional radiotherapy group.

Chemotherapy. The regimens of chemotherapy concurrent with irradiation were cisplatin: $40 \mathrm{mg} / \mathrm{m}^{2}$ weekly for 6 cycles, nedaplatin: $30 \mathrm{mg} / \mathrm{m}^{2}$ weekly for 6 cycles, and cisplatin + fluorouracil: cisplatin $70 \mathrm{mg} / \mathrm{m}^{2}$ + fluorouracil $2.8 \mathrm{~g} / \mathrm{m}^{2} / 96 \mathrm{~h}$ every 3 weeks for 4 cycles in the helical tomotherapy group. The regimens of the chemotherapy were cisplatin: $40 \mathrm{mg} / \mathrm{m}^{2}$ weekly for 6 cycles and nedaplatin: $30 \mathrm{mg} / \mathrm{m}^{2}$ weekly for 6 cycles in the CRT group.

Statistics. Fisher's exact test was used to compare each parameter of patients' characteristics, adverse effect, and overall response between both groups. Progression-free survival (PFS) was analyzed by the Kaplan-Meier method and significance was determined by the log-rank test. $\mathrm{P}<0.05$ was considered to indicate a statistically significant difference. All statistical analyses were carried out using the software JMP Pro version 13 (SAS Institute, Inc., Cary, NC, USA).

\section{Results}

Patients characteristics. The characteristics of patients are shown in Table I. The median age of patients treated with helical tomotherapy was 60 (range, 35-71) and median age of patients treated with CRT was 57 (range, 43-77). The median follow-up period was 27 months (range, 3-46) for the patients treated with helical tomotherapy and 35 months (range, 7-88) for the patients treated with CRT. There were significant differences between the helical tomotherapy group and the CRT group in the methods of chemotherapy $(\mathrm{P}=0.04)$ and radiotherapy $(\mathrm{P}=0.02)$. The single agent regimen of cisplatin was carried out for 14 out of 15 patients in the CRT group. There were significantly more patients who underwent ICBT in the CRT group than in the tomotherapy group. There were no significant differences between the groups with regard to the clinical stage, histological subtypes, or tumor size.

Adverse effect and overall response. There was a significant difference between the helical tomotherapy group and the CRT group in the frequency of $\mathrm{G} 3 / 4$ thrombocytopenia $(\mathrm{P}=0.049)$ (Table II). However, the platelet count spontaneously recovered without transfusion. There were no significant differences between the groups in the frequency of G3/4 neutropenia, diarrhea, or intestine injury (Table II). Patients with G3/4 thrombocytopenia tended to have undergone extended-field irradiation of the para-aortic lymph nodes as an initial treatment, but it was not significant $(\mathrm{P}=0.0675)$. Two out of three patients with G3/4 thrombocytopenia underwent combination chemotherapy consisting of two anticancer agents. In both groups, complete response (CR) or partial response (PR) was achieved by all patients. The rates of CR in the tomotherapy group and the CRT group were 84.6 and $73.3 \%$, respectively. The rates of PR in the tomotherapy group and the CRT group were 15.4 and $26.7 \%$, respectively. There were no statistically significant differences between the helical tomotherapy group and the CRT group in the ratio of CR and PR (Table III).

Clinical outcome. There were no significant differences in PFS ( $\mathrm{P}=0.7826$; Fig. 1) or PFS in the irradiation field $(\mathrm{P}=0.4721$; Fig. 2$)$ between the helical tomotherapy group and the CRT group. In the helical tomotherapy group, 4 patients had recurrence in the irradiation field: Primary lesion in 1, corpus uteri in 1, pelvic lymph node in 1 , and supraclavicular lymph node in 1 . In the helical tomotherapy group, 2 patients had pulmonary metastases. In the helical tomotherapy group, no patient died of cervical cancer. In the CRT group, 3 patients had recurrence of the primary lesion in the irradiation field. In the CRT group, 2 patients had metastases in the para-aortic lymph node, and one patient had hepatic metastasis. 
Table I. Patient characteristics.

\begin{tabular}{|c|c|c|c|}
\hline & $\begin{array}{l}\text { Tomotherapy } \\
\quad(\mathrm{n}=13)\end{array}$ & $\begin{array}{c}\text { CRT } \\
(n=15)\end{array}$ & \\
\hline Variables & $\mathrm{n}(\%)$ & $\mathrm{n}(\%)$ & P-value \\
\hline Age median (range) & $60(35-71)$ & $57(43-77)$ & 0.47 \\
\hline FIGO stage & & & 0.35 \\
\hline IB & $1(7.7)$ & 0 & \\
\hline IIA & 0 & $1(6.7)$ & \\
\hline IIB & $4(30.8)$ & $4(26.7)$ & \\
\hline IIIA & $2(15.4)$ & 0 & \\
\hline IIIB & $4(30.8)$ & $7(46.7)$ & \\
\hline IVA & $1(7.7)$ & $3(20)$ & \\
\hline IVB & $1(7.7)$ & 0 & \\
\hline Histological subtype & & & 0.34 \\
\hline SCC & $13(100)$ & $14(93.3)$ & \\
\hline Adenocarcinoma & 0 & $1(6.7)$ & \\
\hline Tumor size & & & 0.75 \\
\hline$\leqq 40 \mathrm{~mm}$ & $2(15.4)$ & $3(20.0)$ & \\
\hline$>40 \mathrm{~mm}$ & $11(84.6)$ & $12(80.0)$ & \\
\hline Chemotherapy & & & 0.04 \\
\hline CDDP & $7(53.8)$ & $14(93.3)$ & \\
\hline CDGP & $3(23.1)$ & $1(6.7)$ & \\
\hline $\mathrm{CDDP}+5-\mathrm{Fu}$ & $3(23.1)$ & 0 & \\
\hline Radiotherapy & & & 0.02 \\
\hline With ICBT & $9(69.2)$ & $15(100.0)$ & \\
\hline Without ICBT & $4(30.8)$ & 0 & \\
\hline
\end{tabular}

Data are given as n (\%). Fisher's exact test was applied for statistical analysis.CRT, conventional radiotherapy; FIGO, International Federation of Gynecology and Obstetrics; SCC, squamous cell carcinoma; CDDP, cisplatin; CDGP, nedaplatin; 5-Fu, fluorouracil; ICBT, intracavity brachytherapy.

\section{Discussion}

Although IMRT for the treatment of uterine cervical cancer has been described in the practical guidelines by the USA and Japan as well as in the studies mentioned above, there are only a few reports on helical tomotherapy as definitive radiotherapy for cervical cancer. Schwarz et al (6) reported that postoperative external IMRT for cervical cancer delivered with helical tomotherapy and high dose rate brachytherapy with or without chemotherapy was feasible, and had acceptable acute and chronic toxicity. Chang et al (7) described that IMRT delivered with helical tomotherapy and high dose rate brachytherapy with or without chemotherapy for definitive treatment of cervical cancer was feasible, with acceptable levels of chronic toxicity. Jouglar et al (8) found that extended-field helical tomotherapy to the para-aortic nodes was associated with low rates of acute gastrointestinal and genitourinary toxicities, with early survival and locoregional control similar with in other published series. Marnitz et al (9) compared IMRT delivered by helical tomotherapy with conventional
IMRT for primary chemoradiation in cervical cancer patients, and concluded that both helical tomotherapy and conventional IMRT provided optimal treatment of cervical cancer patients, and that the helical tomotherapy technique was significantly favorable regarding conformity, homogeneity, and small bowel sparing.

Problems due to motion of the target and surrounding organs at risk have interfered with IMRT becoming the definitive treatment for cervical cancer. However, helical tomotherapy has the advantage of image guided radiation therapy based on daily 3D megavoltage $\mathrm{CT}$ imaging, and this advantage may overcome the issues with motion of the target and surrounding organs in the definitive treatment of cervical cancer.

In this analysis, there was a significant difference in the method of chemotherapy and the frequency of ICBT. We administered nedaplatin to patients with poor renal function or hydronephrosis in both groups. Cisplatin and fluorouracil were administered to 3 patients in the helical tomotherapy group; one with a bulky stage IIB tumor, one with para-aortic and mediastinal metastases of T2BN1M1, and the other with stage IVB pulmonary metastases. Seven out of 13 patients received paraaortic irradiation and 9 out of 13 patients received ICBT in the helical tomotherapy group. Of the 4 patients without ICBT, 3 had non-para-aortic distant metastases, and one patient had too bulky of a tumor to insert the ICBT tandem in the helical tomotherapy group. In contrast, 4 out of 15 patients received para-aortic irradiation and all patients received ICBT in the CRT group. Although patients with distant metastases, excluding the para-aortic lymph nodes, were not treated with concurrent chemoradiotherapy during CRT, advanced disease with distant metastases is recently treated with concurrent chemoradiotherapy using helical tomotherapy. The disease progression was thought to be related with the differences in chemotherapy and ICBT between the groups, although there were no significant differences in clinical stage between them. There was a significant difference between the helical tomotherapy group and the CRT group in the frequency of G3/4 thrombocytopenia ( $\mathrm{P}=0.049$; Table II). However, the platelet count spontaneously recovered without transfusion. There was a tendency for patients with G3/4 thrombocytopenia to have undergone extended-field irradiation of the para-aortic lymph nodes as an initial treatment, but it was not significant $(\mathrm{P}=0.0675)$. Two out of three patients with $\mathrm{G} 3 / 4$ thrombocytopenia underwent combination chemotherapy with cisplatin and fluorouracil. In the helical tomotherapy group, there were more advanced cases with distant metastases compared with in the CRT group, and disease progression led to the selection of multiagent chemotherapy. In our study, there were no patients with acute or late gastrointestinal adverse effects in the tomotherapy group. Chang et al (7) previously reported that one out of 15 patients who underwent definitive chemoradiotherapy delivered with helical tomotherapy developed a chronic grade 3 gastrointestinal complication. Gandhi et al (5) reported that one out of 22 patients who underwent definitive chemoradiotherapy delivered with IMRT developed an acute $\geq$ grade 3 gastrointestinal complication and no patient developed acute $\geq$ grade 3 genitourinary complications.

In the present study, there were no significant differences in PFS $(\mathrm{P}=0.7826)$ or progression-free rate in the irradiation field 
Table II. Adverse effects.

\begin{tabular}{|c|c|c|c|c|c|}
\hline \multirow[b]{2}{*}{ Variables } & \multicolumn{2}{|c|}{ Tomotherapy $(n=13)$} & \multicolumn{2}{|c|}{ CRT $(n=15)$} & \multirow[b]{2}{*}{ P-value } \\
\hline & Grade 3 & Grade 4 & Grade 3 & Grade 4 & \\
\hline \multicolumn{6}{|l|}{ Acute toxicity } \\
\hline Neutropenia & $6(46.2 \%)$ & 0 & $5(33.3 \%)$ & $1(6.7 \%)$ & 0.74 \\
\hline Thrombocytopenia & $3(23.1 \%)$ & 0 & 0 & 0 & 0.049 \\
\hline Diarrhea & 0 & 0 & $3(20 \%)$ & 0 & 0.088 \\
\hline \multicolumn{6}{|l|}{ Late toxicity } \\
\hline Small/large intestine & 0 & 0 & 0 & $1(6.7 \%)$ & 0.34 \\
\hline
\end{tabular}

Acute toxicity was estimated using common toxicity criteria (version 4.0). Late toxicity was estimated by RTOG/EORTC radiation toxicity criteria. Data are given as n (\%). Fisher's exact test was applied for statistical analysis. CRT, conventional radiotherapy; RTOG, The Radiation Therapy Oncology Group; EORTC, European Organization for Research and Treatment of Cancer.

Table III. Overall response.

\begin{tabular}{lcccc}
\hline & $\begin{array}{c}\text { Tomotherapy } \\
(\mathrm{n}=13)\end{array}$ & & $\begin{array}{c}\text { CRT } \\
(\mathrm{n}=15)\end{array}$ & \\
\cline { 2 - 3 } Response & $\mathrm{n}(\%)$ & & $\mathrm{n}(\%)$ & P-value \\
\hline Complete & $11(84.6)$ & & $11(73.3)$ & 0.468 \\
Partial & $2(15.4)$ & & $4(26.7)$ & \\
\hline
\end{tabular}

Overall response was estimated using the response evaluation criteria in solid tumors guideline (version 1.1). Data are given as $\mathrm{n}(\%)$. Fisher's exact test was applied for statistical analysis. CRT, conventional radiotherapy.

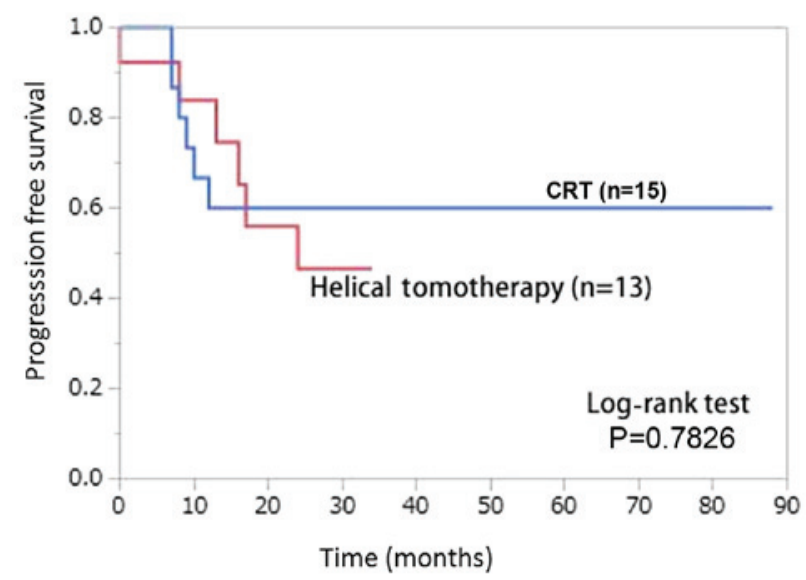

Figure 1. Progression-free survival of patients treated with helical tomotherapy and CRT. CRT, conventional radiotherapy.

$(\mathrm{P}=0.4721)$ between the helical tomotherapy group and the CRT group. The 2-year progression-free rate was 46.6 and $60.0 \%$ in the helical tomotherapy and CRT groups, respectively. The 2-year progression-free rate in the irradiation field was 62.9 and $80.0 \%$ in the helical tomotherapy and CRT groups, respectively. In the

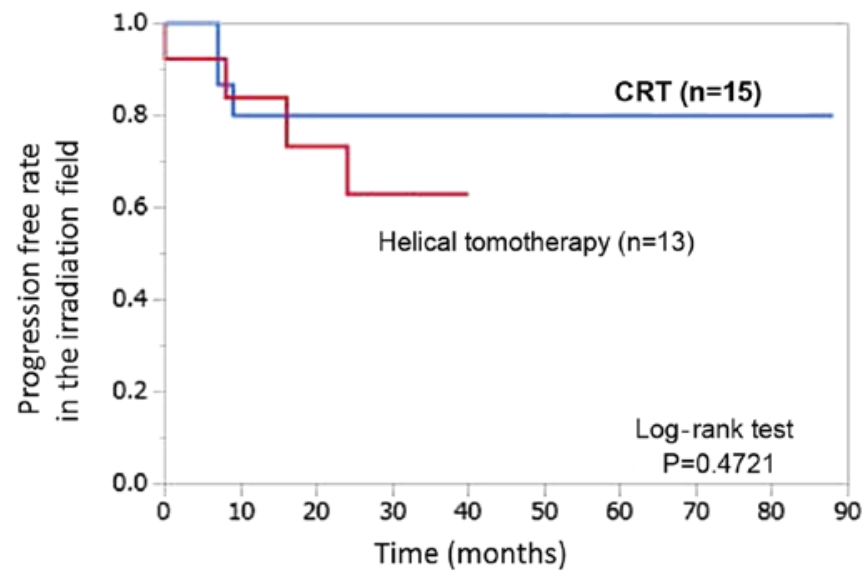

Figure 2. Progression-free rate in the irradiation field of patients treated with helical tomotherapy or CRT. CRT, conventional radiotherapy.

helical tomotherapy group, 6 out of 13 patients had recurrence; two with para-aortic metastases, two with distant metastases not in the para-aortic lymph nodes, and the remaining two had bulky stage IIB tumors before the start of the therapy. In the CRT group, 6 out of 15 patients had recurrence; three had stage IIIB tumors, two had stage IVA tumors, and the other had a bulky stage IIB tumor before the start of the therapy. In both groups, careful observation was necessary in terms of recurrence of stage III, IV, and bulky IIB tumors. There were no significant differences in the prognosis between both groups, but the helical tomotherapy group included more patients with distant metastases. If those with distant metastases, including para-aortic lymph nodes, are excluded from the helical tomotherapy group, the prognosis based on the data may improve.

In conclusion, adverse events from concurrent chemoradiotherapy using helical tomotherapy were acceptable and clinically controllable. Helical tomotherapy is efficient during concurrent chemoradiotherapy for advanced cervical cancer. Prospective studies involving more patients for longer follow-up periods are needed to assess the difference in oncological outcome and toxicity between helical tomotherapy and conventional radiotherapy. 


\section{Acknowledgements}

The authors thank Dr Takahiro Chiba (Department of Radiation Oncology, Wakayama Medical University) for his assistance of data management on radiotherapy planning.

\section{Funding}

No funding was received.

\section{Availability of data and materials}

The datasets analyzed during the current study are available from the corresponding author on reasonable request.

\section{Authors' contributions}

All authors participated in the conception and design of the study. YM, YT, TY, MM, NS, NO and SM obtained the data. YM analyzed the data and drafted the manuscript. KI revised the manuscript prior to submission. In addition, KI and YM were major contributors in designing the study. All authors have read and approved the final version of the manuscript.

\section{Ethics approval and consent to participate}

The current study was approved by the ethical committee of Wakayama Medical University (reference no. 2337). Patient consent was also obtained.

\section{Patient consent for publication}

The patients provided written informed consent for the publication of any associated data and accompanying images.

\section{Competing interests}

The authors declare that they have no competing interests.

\section{References}

1. Keys HM, Bundy BN, Stehman FB, Muderspach LI, Chafe WE, Suggs CL III, Walker JL and Gersell D: Cisplatin, radiation and adjuvant hysterectomy compared with radiation and adjuvant hysterectomy for bulky stage IB cervical carcinoma. N Engl J Med 340: 1154-1161, 1999.

2. Tseng CJ, Chang CT, Lai CH, Soong YK, Hong JH, Tang SG and Hsueh S: A randomized trial of concurrent chemoradiotherapy versus radiotherapy in advanced carcinoma of the uterine cervix. Gynecol Oncol 66: 52-58, 1997.

3. Peters WA III, Liu PY, Barrett RJ II, Stock RJ, Monk BJ, Berek JS, Souhami L, Grigsby P, Gordon W Jr and Alberts DS: Concurrent chemotherapy and pelvic radiation therapy compared with pelvic radiation therapy alone as adjuvant therapy after radical surgery in high-risk early-stage cancer of the cervix. J Clin Oncol 18: 1606-1613, 2000.

4. Kirwan JM, Symonds P, Green JA, Tierney J, Collingwood M and Williams CJ: A systematic review of acute and late toxicity of concomitant chemoradiation for cervical cancer. Radiother Oncol 68: 217-226, 2003.

5. Gandhi AK, Sharma DN, Rath GK, Julka PK, Subramani V, Sharma S, Manigandan D, Laviraj MA, Kumar S and Thulkar S: Early clinical outcomes and toxicity of intensity modulated versus conventional pelvic radiation therapy for locally advanced cervix carcinoma: A prospective randomized study. Int J Radiat Oncol Biol Phys 87: 542-548, 2013.

6. Schwarz JK, Wahab S and Grigsby PW: Prospective phase I-II trial of helical tomotherapy with or without chemotherapy for postoperative cervical cancer patients. Int J Radiat Oncol Biol Phys 81: 1258-1263, 2011.

7. Chang AJ, Richardson S, Grigsby PW and Schwarz JK: Split-field helical tomotherapy with or without chemotherapy for definitive treatment of cervical cancer. Int J Radiat Oncol Biol Phys 82: 263-269, 2012.

8. Jouglar E, Thomas L, de la Rochefordière A, Noël G, Le Blanc-Onfroy M, Delpon G, Campion L and Mahé MA: Toxicity and early clinical outcomes in cervical cancer following extended field helical tomotherapy to para-aortic lymph nodes. Cancer Radiother 20: 794-800, 2016.

9. Marnitz S, Lukarski D, Köhler C, Wlodarczyk W, Ebert A, Budach V, Schneider A and Stromberger C: Helical tomotherapy versus conventional intensity-modulated radiation therapy for primary chemoradiation in cervical cancer patients: An intraindividual comparison. Int J Radiat Oncol Biol Phys 81: 424-430, 2011. 